\title{
Assessment of Genetic Diversity for Some Egyptian Wheat Varieties based on Morphological Characters and SSR Markers
}

\author{
Mahmoud A. El-Rawy \\ Department of Genetics, Faculty of Agriculture, Assiut University, Assiut, Egypt \\ Corresponding author email: mabosuud2020@aun.edu.eg
}

Received on: 28/7/2020

Accepted on: 3/9/2020

\begin{abstract}
The genetic diversity estimates can be useful in important genotypes selection for plant breeders. Eight durum and three bread wheat cultivars were used to study and compare morphological traits with molecular study using SSR technique based genetic diversity estimates. Significant and highly significant differences among wheat genotypes were observed for all traits except flag leaf attitude and outer glume pubescence. Highly significant differences were obtained between durum and bread wheat genotypes for most studied traits. Moderate to low broad-sense heritability estimates were obtained for morphological studied traits. Positive and high significant correlation was found for ear density with foliage color $(\mathrm{r}=0.51)$, waxiness of flag leaf sheath $(\mathrm{r}=0.57)$, waxiness of peduncle $(\mathrm{r}=0.60)$ and peduncle length $(\mathrm{r}=0.53)$, whereas a negative correlation was found between ear density and plant height $(\mathrm{r}=-0.52, \mathrm{P}<0.01)$. However, waxiness of flag leaf sheath was positively correlated with ear waxiness $(\mathrm{r}=0.53, \mathrm{P}<0.01)$, waxiness of peduncle $(\mathrm{r}=0.56, \mathrm{P}<0.01)$, and Peduncle length $(\mathrm{r}=$ $0.43, \mathrm{P}<0.05)$. The higher polymorphism $(90.63 \%)$ was found in the B genome than in the A genome (81.97\%). Single marker analysis showed that 11 SSR markers were significantly associated with phenotypic traits, including Xgwm111-2B associated with waxiness of peduncle. A significant but low correlation $(r=0.25)$ was found between the dissimilarity matrix generated from the phenotypic data and that obtained from the SSR markers, suggesting that the characterization based on agromorphological traits and SSR markers will be a useful tool to the breeders for selection of genotypes with appropriate.
\end{abstract}

KEYWORDS: Durum wheat, Bread wheat, SSR markers, Waxiness, Polymorphism.

\section{INTRODUCTION}

Wheat is the most widely grown crop in the world, adapted to a wide range of environments, and considered as a staple source of nutrients for around $40 \%$ of the world's population (Giraldo et al., 2019).wheat is the most important grain crop in Egypt and plays a vital role in Egypt's economy as a strategic crop. In Egypt, There is a large gap between the production of this crop and its consumption. Therefore, Egypt remains the largest wheat importer in the world, where wheat imports for the 2019/20 marketing year were estimated at 12.5 million tons, about $15 \%$ above the average of the last five years (FAOSTAT, 2020). So, it is important to improve this crop to overcome this problem.

The genetic diversity among plant species offers prospects for the improvement of plant characteristics, and it is an important source for any successful breeding program. Therefore, assessment of the genetic diversity is necessary for the effective exploitation of genetic resources in breeding programs (Khan et al., 2015). Moreover, differences in the genetic components of the traits can be applied as a new source of variation in other breeding programs for wheat improvement (Khodadadi et al., 2011). Determination of the genetic diversity and the relationships among genotypes is also of great importance for identifying the appropriate parents in breeding programs (Ghasemi et al., 2019).

The methods for detecting and assessing the genetic diversity among genotypes have extended from the analysis of discrete morphological to biochemical and molecular traits (Khaled et al., 2015). Characterization of wheat genotypes based on qualitative and quantitative agro-morphological traits could be helpful in constructing breeding populations and implementing selection strategies (Aghaee et al., 2010). Furthermore, assessment of the genetic variability using molecular markers has been used for understanding the genomic constitution in plants genome and categorizing genes responsible for important traits (Khan et al., 2015). Therefore, the genetic diversity in wheat was successfully assessed using morphological traits (EL-Rawy and Youssef, 


\section{Mahmoud A. El-Rawy., 2020}

2014, Hassan, 2016) and molecular markers (Salem et al., 2015, Hassan, 2016).

Although, morphological traits can be used for assessing the genetic diversity, they are often influenced by the environment (Hassan, 2016). Therefore, the use of molecular markers for assessment of the genetic diversity is receiving much attention from wheat breeders (Salem et al., 2015). In addition, molecular markers provided the opportunity for determining inter and intra-species genetic relationships (Gostimsky et al. 2005). Therefore, characterization based on DNA polymorphism using molecular markers is more efficient and accurate. Numerous PCR-based molecular markers were developed to assess the genetic diversity among different genotypes. These marker systems are different in their technical principles as well as the amount of polymorphism (Powell et al. 1995).

Microsatellites or simple sequence repeats (SSRs) are characterized by a high level of polymorphism, chromosome-specific, multiallelic and distributed over the genome. These characteristics allow SSR markers to discriminate among cultivars and even among closely related wheat breeding lines (Mantovani et al., 2008; Salem et al., 2015). Therefore, SSR markers have been long used for several purposes including genome mapping, physical mapping, gene tagging and genetic diversity estimates (Wang et al., 2007). Several hundred SSRs have been developed for the A, B, and D genomes of wheat (Mantovani et al., 2008), and used in the association analysis and linkagebased studies for mapping genes or quantitative trait loci (QTL) controlling important traits (Neale and Savolainen 2004).

Clustering genotypes based on similar characteristics could provide valuable information for selecting the better performing lines in breeding programs. Thus, cluster analysis has been widely used for assessment of the genetic diversity and grouping wheat genotypes based on phenotypic data and molecular markers (El-Rawy and Hassan, 2014; Arain et al., 2018; Jamali et al., 2020).

The aims of the present study were to evaluate eight durum and three bread wheat cultivars for several morphological traits; identify molecular markers associated with studied traits to be used in breeding programs and assess the genetic diversity among the tested plant genotypes based on the results of phenotypic traits and SSR markers.

\section{MATERIALS AND METHODS}

\subsection{Plant materials and morphological characters}

In the present study, eight Egyptian durum (Triticum durum Desf.) and three bread (T. aestivum L.) wheat cultivars was chosen for studying the morphological characters as shown in Table (1). The field experiment was conducted at the Experimental Farm of Faculty of Agriculture, Assiut University, Egypt during two growing seasons of 2017/18 and 2018/19.

Seeds of the tested plant genotypes were cultivated on normal sowing dates. The field experiment was designed as a randomized complete block design (RCBD) with three replications. The experimental plot consisted of 6 rows, $20 \mathrm{~cm}$ apart, and $3.5 \mathrm{~m}$ long (plot area $=4.2 \mathrm{M}^{2}$ ). The cultivars under investigation were phenotyped using some morphological characters used for distinctness, uniformity and stability (DUS). The morphological descriptors were 17 characters. Scoring values for each state of selected descriptors were given discrete number value to generate numerical dataset (Table 2).

\subsection{Simple sequence repeats (SSRs) technique}

The simple sequence repeats (SSRs) technique was carried out at the Department of Genetics, Faculty of Agriculture, Assuit University, Egypt. Twenty one wheat microsatellites or SSR primer pairs were selected and used for screening the studied genotypes (Table 3 ). The A, B and D wheat genomes were covered by a primer pair for each chromosome. The total genomic DNA of each cultivar was extracted by Cetyl trimethyl ammonium bromide (CTAB) method (Murray and Thompson 1980).

Twenty one Primer sequences were used and performed by GrainGenes Database (http://wheat.pw.usda.gov) in the present study. The PCR conditions and its thermal cycle were carried out according to a SensoQuest LabCycler (SensoQuest $\mathrm{GmbH}$, Göttingen, Germany). The PCR products were separated on $2.5 \%$ agarose gels in $0.5 \times$ TBE buffer. A 100bp DNA Ladder was used to estimate the molecular size of the amplified DNA fragments. The percentage of polymorphism obtained by each marker was calculated by dividing the number of polymorphic bands with the total number of amplified bands. The polymorphic information content (PIC) was calculated for each marker using the formula described by RoldanRuiz et al. (2000) to investigate the suitability of each marker and assess the genetic diversity among the studied plant genotypes. Also, Marker index (MI) and Resolving power (Rp) of each RAPD primer were 
Scientific Journal of Agricultural Sciences 2 (1): 00-00, 2020

Table 1. Names, code and pedigree of eight durum and three bread wheat cultivars.

\begin{tabular}{|c|c|c|c|}
\hline & Name & Code & Pedigree \\
\hline \multirow{10}{*}{ 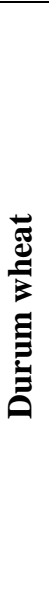 } & Bani Sueif 1 & G1 & JORI69(SIB)/(SIB)ANHINGA//(SIB)FLAMINGO \\
\hline & Bani Sueif 4 & G2 & RoK"S"/Mexi75/a/"S"//Ruff"S"/FG"S"/3/Mexi 75 \\
\hline & Bani Sueif 5 & G3 & DIPPER-2/ BUCHEN-3 \\
\hline & Bani Sueif 6 & G4 & BOOMER-21/BUSCA-3 \\
\hline & & & CBC509CHILE//sooty_9/RASCON_37/9/USDA595/3/D67.3/RABI \\
\hline & Dam suen & Us & //CRA/4/ALO/5/HUI/YAV_1/6/ARDENTE/7/HUI/YAV79/8/POD_9 \\
\hline & Sohag 3 & G6 & MEXICALI-75/MAGHREBI-72//S-179/DURUM-6 \\
\hline & Sohag 4 & G7 & Ajaia-16//Hora/Jor/3/Gan/4/Zar/5/Souk-7/6/Stot//Altar84/aLD \\
\hline & & & TRN//21563/AA/3/BD2080/4/BD2339/5/RASCON37/Tarro 2//RASCON \\
\hline & Nonag & 00 & 3/6/Auk/Gull//Green \\
\hline \multirow{4}{*}{$\frac{\vec{J}}{\underset{D}{ \pm}}$} & Gemmeiza7 & G9 & CMH74 A. 630/5x//Seri 82/3/Agent CGM 4611-2GM-3GM-1GM-0GM \\
\hline & Gemmeiza9 & G10 & Ald"S"/Huac"S"//CMH74A.630/5x CGM4583-5GM-1GM-0GM \\
\hline & Misr 1 & G11 & OASIS/SKAUZ//4*BCN/3/2*PASTOR. CMSS00Y01881T -050M-030Y \\
\hline & & & -030M-030WGY-33M0Y--0EGY \\
\hline
\end{tabular}

calculated according to Powell et al., (1996) and Prevost \&Wilkinson (1999), respectively. .

\subsection{Statistical analysis for morphological traits and SSR data}

The differences between means were tested by Fisher's Least Significant Difference (LSD) at 0.05 and 0.01 levels of probability. Combined analyis of variance was used to test the significance of differences among genotypes (G) and years (E), and the significance of $\mathrm{G} \times \mathrm{E}$ interaction for each trait. The broad-sense heritability $\left(h^{2} \mathrm{~B}\right)$ of a trait was computed using the formula described by Nyquist (1991). The phenotypic correlations among the studied traits were measured by Pearson's correlation coefficients.

Cluster analysis of wheat genotypes based phenotypic data was conducted using Standardized Euclidean distance matrix with the unweighted pair group method based on arithmetic averages (UPGMA) by MVSP version 3.22 software (Kovach Computing Services). The genetic distance matrix based on SSR markers was conducted and UPGMA-dendrogram was performed according to Nei and Li's coefficient using MVSP version 3.22. In order to investigate the association between the SSR markers and the studied traits, single marker analysis using linear regression was conducted by Microsoft Excel.

\section{RESULTS}

The mean performance of the studied genotypes for Growth habit $(\mathrm{GH})$, Auricles coloration pigment (CLAR), Flag leaf attitude (FLAT), Waxiness of flag leaf sheath (WSH), Waxiness of peduncle (W. Ped), Ear shape in profile (Ear Shape), Ear orientation (Ear Orint), Foliage color (FCL), Plant height (PLHT), Ear waxiness (W. Ear), Flag leaf width (FLW), Flag leaf length (FLL), Leaf blade waxiness (WBL), Peduncle length (Ped. L), Ear density (Ear. Dens), Hairs of auricles (HRAR), Outer glume pubescence (Out. Gl. Pub) during the two seasons is presented in Table (4). Means of GH, FCL, CLAR, WSH, Ped L and Ear Den in durum wheat $(3.46,4.92,4.58,6.38,6.08$ and 7.21, respectively) were significantly higher than those obtained for bread wheat $(2.33,3.00,2.78,4.89,4.56$ and 5.11, respectively). Unlike, means HRAR, FLW and PLHT in bread wheat $(5.56,8.78$ and 6.22, respectively) were significantly higher than those observed for durum wheat $(4.29,7.67$, and 5.13, respectively). While, nonsignificant differences were found between durum and bread wheat genotypes for the remaining traits. Out of eight durum wheat genotypes tested, the means of FCL, Ped. L and Out.G1.Pub in G1, G2, G3, G4 and G5 genotypes (Bani Sueif) were higher than those of G6, G7 and G8 genotypes (Sohag) by 47.38, 24.11 and 34.19\%, respectively. Unlike, the means of CLAR were greater in G6, G7 and G8 sohag genotypes than those found in 
Mahmoud A. El-Rawy., 2020

G1, G2, G3, G4 and G5 Bani Sueif genotypes by $45.11 \%$. 
Scientific Journal of Agricultural Sciences 2 (1): 00-00, 2020

Table 2. Morphological characters and their numerical scores in 11 Egyptian wheat cultivars.

\begin{tabular}{|c|c|c|c|c|c|}
\hline Characters & Abbrev. & Descriptors & Characters & Abbrev. & Descriptors \\
\hline Growth habit & $\mathrm{GH}$ & $\begin{array}{c}1 \text { Erect } \\
3 \text { Semi-erect } \\
5 \text { Intermediate } \\
7 \text { Semi- spreading } \\
9 \text { prostrate }\end{array}$ & Flag leaf width & FLW & $\begin{array}{c}1 \text { Narrow }(<1.5) \\
5 \text { Medium }(1.5-2) \\
9 \text { Broad }(>2.0)\end{array}$ \\
\hline Foliage color & FCL & $\begin{array}{l}1 \text { Pale green } \\
5 \text { Green } \\
9 \text { dark green }\end{array}$ & $\begin{array}{l}\text { Flag leaf } \\
\text { length }\end{array}$ & FLL & $\begin{array}{c}1 \text { Short }(<20) \\
5 \text { Medium }(20-30) \\
9 \text { Long }(>30)\end{array}$ \\
\hline $\begin{array}{l}\text { Hairs of } \\
\text { auricles }\end{array}$ & HRAR & $\begin{array}{l}3 \text { Absent } \\
5 \text { Medium } \\
7 \text { Strong } \\
\end{array}$ & $\begin{array}{c}\text { Peduncle } \\
\text { length }\end{array}$ & Ped. L & $\begin{array}{c}1 \text { Short }(<30) \\
5 \text { Medium }(30-50) \\
9 \text { Long }(>50)\end{array}$ \\
\hline $\begin{array}{c}\text { Auricles } \\
\text { coloration } \\
\text { pigment }\end{array}$ & CLAR & $\begin{array}{l}1 \text { Absent } \\
5 \text { Medium } \\
9 \text { Strong }\end{array}$ & Plant height & PLHT & $\begin{array}{c}1 \text { Very short }(<75 \\
\mathrm{cm}) \\
3 \text { Short }(75.1-90 \mathrm{~cm}) \\
5 \text { Medium }(90.1- \\
105 \mathrm{~cm}) \\
7 \text { Long }(105.1-120 \\
\mathrm{cm}) \\
9 \text { Very Long }(>120 \\
\mathrm{cm})\end{array}$ \\
\hline $\begin{array}{l}\text { Flag leaf } \\
\text { attitude }\end{array}$ & FLAT & $\begin{array}{c}1 \text { Erect } \\
3 \text { Semi-erect } \\
5 \text { drooping } \\
\end{array}$ & $\begin{array}{l}\text { Outer glume } \\
\text { pubescence }\end{array}$ & $\begin{array}{l}\text { Out. Gl. } \\
\text { Pub }\end{array}$ & $\begin{array}{c}3 \text { Absent } \\
5 \text { Medium } \\
7 \text { Strong } \\
\end{array}$ \\
\hline $\begin{array}{l}\text { Waxiness of } \\
\text { flag leaf } \\
\text { sheath }\end{array}$ & WSH & $\begin{array}{c}1 \text { Absent } \\
3 \text { Weak } \\
5 \text { Medium } \\
7 \text { Strong } \\
9 \text { Very strong }\end{array}$ & Ear density & Ear. Dens & $\begin{array}{l}1 \text { Very lax } \\
3 \text { Lax } \\
5 \text { Medium } \\
7 \text { Dense } \\
9 \text { Very dense }\end{array}$ \\
\hline $\begin{array}{l}\text { Leaf blade } \\
\text { waxiness }\end{array}$ & WBL & $\begin{array}{c}1 \text { Absent } \\
3 \text { Weak } \\
5 \text { Medium } \\
7 \text { Strong } \\
9 \text { Very strong }\end{array}$ & $\begin{array}{c}\text { Ear } \\
\text { orientation }\end{array}$ & Ear Orint & $\begin{array}{c}3 \text { Erect } \\
5 \text { Semi-erect } \\
7 \text { Dropping }\end{array}$ \\
\hline Ear waxiness & W. Ear & $\begin{array}{c}1 \text { Absent } \\
3 \text { Weak } \\
5 \text { Medium } \\
7 \text { Strong } \\
9 \text { Very strong }\end{array}$ & $\begin{array}{c}\text { Ear shape in } \\
\text { profile }\end{array}$ & Ear SH & $\begin{array}{c}1 \text { Tapering } \\
3 \text { Parallel sided } \\
5 \text { Semi-clavate } \\
7 \text { Clavate } \\
9 \text { Fusiform } \\
\end{array}$ \\
\hline $\begin{array}{l}\text { Waxiness of } \\
\text { peduncle }\end{array}$ & W. Ped & $\begin{array}{c}1 \text { Absent } \\
3 \text { Weak } \\
5 \text { Medium } \\
7 \text { Strong } \\
9 \text { Very strong }\end{array}$ & & & \\
\hline
\end{tabular}




\section{Mahmoud A. El-Rawy., 2020}

Table 3. Names, chromosomal location (CL), sequences, and annealing temperature (An.) of SSR markers used in the study.

\begin{tabular}{|c|c|c|c|c|}
\hline Marker & $\mathbf{C L}$ & Forward primer & Reverse primer & An. \\
\hline Xgwm33 & $1 \mathrm{~A}$ & 5' GGAGTCACACTTGTTTGTGCA 3' & 5' CACTGCACACCTAACTACCTGC 3' & $60 \mathrm{C}^{\mathrm{c}}$ \\
\hline Xgwm95 & $2 \mathrm{~A}$ & 5' GATCAAACACACACCCCTCC 3' & 5' AATGCAAAGTGAAAAACCCG 3' & $60 \mathrm{C}^{\mathrm{c}}$ \\
\hline Xgwm155 & $3 \mathrm{~A}$ & 5' CAATCATTTCCCCCTCCC 3' & 5' AATCATTGGAAATCCATATGCC 3' & $60 \mathrm{C}^{\mathrm{c}}$ \\
\hline Xgwm160 & $4 \mathrm{~A}$ & 5' TTCAATTCAGTCTTGGCTTGG 3' & 5' CTGCAGGAAAAAAAGTACACCC 3' & $55 \mathrm{C}^{\mathrm{c}}$ \\
\hline Xgwm186 & $5 \mathrm{~A}$ & 5' GCAGAGCCTGGTTCAAAAAG 3' & 5' CGCCTCTAGCGAGAGCTATG 3' & $60 \mathrm{C}^{\mathrm{c}}$ \\
\hline Xgwm459 & $6 \mathrm{~A}$ & 5' ATGGAGTGGTCACACTTTGAA 3' & 5' AGCTTCTCTGACCAACTTCTCG 3' & $55 \mathrm{C}^{\mathrm{c}}$ \\
\hline Xgwm63 & $7 \mathrm{~A}$ & 5' TCGACCTGATCGCCCCTA 3' & 5' CGCCCTGGGTGATGAATAGT 3' & $60 \mathrm{C}^{\mathrm{c}}$ \\
\hline Xgwm18 & $1 \mathrm{~B}$ & 5' GGTTGCTGAAGAACCTTATTTAGG 3' & 5' TGGCGCCATGATTGCATTATCTTC 3' & $50 \mathrm{C}^{\mathrm{c}}$ \\
\hline Xgwm111 & $2 \mathrm{~B}$ & 5'GTTGCACGACCTACAAAGCA 3' & 5'ATCGCTCACTCACTATCGGG 3' & $55 \mathrm{C}^{\mathrm{c}}$ \\
\hline Xgwm389 & 3B & 5' ATCATGTCGATCTCCTTGACG 3' & 5' TGCCATGCACATTAGCAGAT 3' & $60 \mathrm{C}^{\mathrm{c}}$ \\
\hline Xgwm513 & 4B & 5' ATCCGTAGCACCTACTGGTCA 3' & 5' GGTCTGTTCATGCCACATTG 3' & $60 \mathrm{C}^{\mathrm{c}}$ \\
\hline Xgwm408 & $5 \mathrm{~B}$ & 5' TCGATTTATTTGGGCCACTG 3' & 5' GTATAATTCGTTCACAGCACGC 3' & $55 \mathrm{C}^{\mathrm{c}}$ \\
\hline Xgwm626 & $6 \mathrm{~B}$ & 5' GATCTAAAATGTTATTTTCTCTC 3' & 5' TGACTATCAGCTAAACGTGT 3' & $50 \mathrm{C}^{\mathrm{c}}$ \\
\hline Xgwm577 & 7B & 5' ATGGCATAATTTGGTGAAATTG 3' & 5' TGTTTCAAGCCCAACTTCTATT 3' & $55 \mathrm{C}^{\mathrm{c}}$ \\
\hline Xgwm458 & $1 \mathrm{D}$ & 5' TTCGCAATGTTGATTTGGC 3' & 5' TTCGCAATGTTGATTTGGC 3' & $60 \mathrm{C}^{\mathrm{c}}$ \\
\hline Xgwm261 & $2 \mathrm{D}$ & 5' CTCCCTGTACGCCTAAGGC 3' & 5' CTCGCGCTACTAGCCATTG 3' & $55 \mathrm{C}^{\mathrm{c}}$ \\
\hline Xgwm3 & $3 \mathrm{D}$ & 5' AATATCGCATCACTATCCCA 3' & 5' AATATCGCATCACTATCCCA 3' & $55 \mathrm{C}^{\mathrm{c}}$ \\
\hline Xgwm165 & 4D & 5' TGCAGTGGTCAGATGTTTCC 3' & 5' CTTTTCTTTCAGATTGCGCC 3' & $60 \mathrm{C}^{\circ}$ \\
\hline Xgwm190 & $5 \mathrm{D}$ & 5' GTGCTTGCTGAGCTATGAGTC 3' & 5' GTGCCACGTGGTACCTTTG 3' & $60 \mathrm{C}^{\mathrm{c}}$ \\
\hline Xgwm325 & $6 \mathrm{D}$ & 5' тTTCTTCTGTCGTTCTCTTCCC 3' & 5' TTTTTACGCGTCAACGACG 3' & $60 \mathrm{C}^{\mathrm{c}}$ \\
\hline Xgwm437 & 7D & 5' GATCAAGACTTTTGTATCTCTC 3' & 5' GATGTCCAACAGTTAGCTTA 3' & 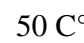 \\
\hline
\end{tabular}


Scientific Journal of Agricultural Sciences 2 (1): 00-00, 2020

Table 4. Two seasons means of studied traits for durum and bread wheat genotypes.

\section{Genotypes}

\begin{tabular}{|c|c|c|c|c|c|c|c|c|c|c|c|c|c|}
\hline \multirow[b]{2}{*}{ 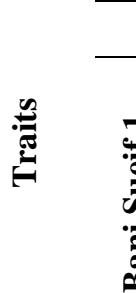 } & \multicolumn{8}{|c|}{ Durum wheat } & \multicolumn{4}{|c|}{ Bread wheat } & \multirow{2}{*}{ 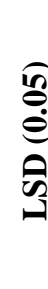 } \\
\hline & 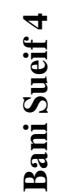 & 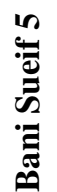 & 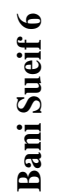 & 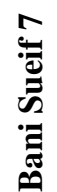 & 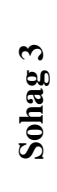 & 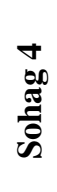 & 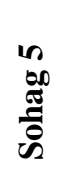 & 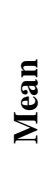 & 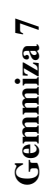 & 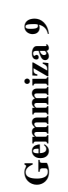 & $\stackrel{\bar{n}}{i}$ & $\sum_{\Sigma}^{\tilde{\Xi}}$ & \\
\hline
\end{tabular}

\begin{tabular}{|c|c|c|c|c|c|c|c|c|c|c|c|c|c|c|}
\hline GH & 3.00 & 1.67 & 5.33 & 2.33 & 4.67 & 3.67 & 3.33 & 3.67 & 3.46 & 2.00 & 2.67 & 2.33 & 2.33 & 1.16 \\
\hline FCL & 3.00 & 5.67 & 6.33 & 7.00 & 6.33 & 4.33 & 3.67 & 3.00 & 4.92 & 3.67 & 3.67 & 1.67 & 3.00 & 1.60 \\
\hline HRAR & 4.33 & 3.33 & 3.67 & 5.33 & 5.00 & 3.33 & 5.00 & 4.33 & 4.29 & 5.33 & 6.33 & 5.00 & 5.56 & 0.91 \\
\hline CLAR & 1.67 & 3.67 & 5.00 & 5.00 & 4.33 & 5.00 & 7.00 & 5.00 & 4.58 & 1.67 & 4.33 & 2.33 & 2.78 & 1.45 \\
\hline FLAT & 2.00 & 1.67 & 1.67 & 2.33 & 2.67 & 2.00 & 1.67 & 2.00 & 2.00 & 2.67 & 2.00 & 1.67 & 2.11 & 0.90 \\
\hline WSH & 4.00 & 6.33 & 6.33 & 7.33 & 7.33 & 6.67 & 7.00 & 6.00 & 6.38 & 4.67 & 4.33 & 5.67 & 4.89 & 0.96 \\
\hline WBL & 3.33 & 5.33 & 4.00 & 5.00 & 6.00 & 4.00 & 6.00 & 5.67 & 4.92 & 5.33 & 3.67 & 4.33 & 4.44 & 1.14 \\
\hline $\begin{array}{l}\text { W. } \\
\text { Ear }\end{array}$ & 4.33 & 6.33 & 4.33 & 7.00 & 6.33 & 4.33 & 7.00 & 5.33 & 5.63 & 5.00 & 5.33 & 5.00 & 5.11 & 0.89 \\
\hline $\begin{array}{l}\text { W. } \\
\text { Ped }\end{array}$ & 5.00 & 5.00 & 6.67 & 7.33 & 6.00 & 4.67 & 6.33 & 6.00 & 5.88 & 4.33 & 5.00 & 6.33 & 5.22 & 0.79 \\
\hline FLW & 5.67 & 9.00 & 9.00 & 7.00 & 7.67 & 9.00 & 8.33 & 5.67 & 7.67 & 9.00 & 9.00 & 8.33 & 8.78 & 1.16 \\
\hline FLL & 7.00 & 8.33 & 5.67 & 5.67 & 5.00 & 8.33 & 8.33 & 5.00 & 6.67 & 7.00 & 5.00 & 8.33 & 6.78 & 1.30 \\
\hline Ped. L & 5.00 & 6.33 & 7.00 & 6.33 & 7.67 & 6.33 & 5.00 & 5.00 & 6.08 & 3.67 & 5.00 & 5.00 & 4.56 & 1.02 \\
\hline PLHT & 5.33 & 5.33 & 5.00 & 5.33 & 5.33 & 4.67 & 5.00 & 5.00 & 5.13 & 6.33 & 6.67 & 5.67 & 6.22 & 0.70 \\
\hline $\begin{array}{l}\text { Out. } \\
\text { Gl. } \\
\text { Pub. }\end{array}$ & 5.67 & 5.00 & 5.33 & 4.00 & 5.33 & 4.33 & 4.00 & 3.00 & 4.58 & 5.00 & 4.33 & 4.67 & 4.67 & 1.14 \\
\hline $\begin{array}{l}\text { Ear } \\
\text { Den }\end{array}$ & 6.67 & 6.33 & 7.00 & 8.00 & 8.00 & 6.67 & 7.33 & 7.67 & 7.21 & 4.33 & 6.33 & 4.67 & 5.11 & 0.83 \\
\hline $\begin{array}{l}\text { Ear } \\
\text { Orint }\end{array}$ & 3.33 & 6.33 & 5.00 & 4.67 & 5.00 & 5.67 & 5.33 & 5.67 & 5.13 & 6.33 & 4.00 & 5.00 & 5.11 & 1.14 \\
\hline Ear Sh & 3.33 & 2.00 & 4.00 & 4.00 & 4.33 & 4.33 & 2.33 & 4.67 & 3.63 & 1.00 & 3.00 & 5.00 & 3.00 & 0.99 \\
\hline
\end{tabular}

Growth habit (GH), Auricles coloration pigment (CLAR), Flag leaf attitude (FLAT), Waxiness of flag leaf sheath (WSH), Waxiness of peduncle (W. Ped), Ear shape in profile (Ear Shape), Ear orientation (Ear Orint), Foliage color (FCL), Plant height (PLHT), Ear waxiness (W. Ear), Flag leaf width (FLW), Flag leaf length (FLL), Leaf blade waxiness (WBL), Peduncle length (Ped. L), Ear density (Ear. Dens), Hairs of auricles (HRAR), Outer glume pubescence (Out. Gl. Pub). 


\section{Mahmoud A. El-Rawy., 2020}

\subsection{Analysis of variance and heritability estimates}

The combined analysis of variance (Table 5) revealed highly significant differences among the two growing seasons (S) for GH, FCL, HRAR, WBL, W. Ped and Ear Den. Significant $(P<0.05)$ or highly significant differences $(P<0.01)$ among wheat genotypes $(\mathrm{G})$ were observed for all the traits except FLAT and Out.Gl.Pub. Highly significant $(P<0.01)$ differences were obtained between durum and bread wheat genotypes for GH, FCL, HRAR, CLAR, WSH, FLW, Ped. L, PLHT and Ear Den., while significant
$(P<0.05)$ differences were obtained for W. Ped. Highly significant $\mathrm{G} \times \mathrm{S}$ interactions $(P<0.01)$ were observed for HRAR, CLAR, W. Ear, W. Ped and Ped. L, whereas significant interactions $\mathrm{G} \times \mathrm{S}(P<0.05)$ were obtained for $\mathrm{GH}$ and Out.Gl.Pub. Moderate broad-sense heritability estimates were obtained for WSH (0.45), FLW (0.52), and FLL (0.47), Ear Den (0.50) and Ear Sh (0.52), whereas low heritability was found for GH (0.17), FCL (0.29), HRAR (0.14), CLAR (0.14), FLAT (0.01), WBL (0.15), W. Ear (0.05), W. Ped (0.25), Ped. L (0.08), PLHT (0.23), Out.Gl.Pub (0.01) and Ear Orint (0.17) (Table 5).

Table 5. The combined analysis of variance and broad-sense heritability for the studied traits.

\begin{tabular}{|c|c|c|c|c|c|c|c|c|c|c|}
\hline \multirow[b]{2}{*}{ S. O. V. } & \multirow[b]{2}{*}{ df } & \multicolumn{9}{|c|}{ Mean squares } \\
\hline & & GH & FCL & HRAR & CLAR & FLAT & WSH & WBL & $\begin{array}{l}\text { W. } \\
\text { Ear }\end{array}$ & W. Ped \\
\hline Seasons & 1 & $21.88^{* *}$ & $29.33 * *$ & $8.73 * *$ & 8.73 & 3.88 & 3.88 & $7.33^{*}$ & 0.24 & $7.33 * *$ \\
\hline R./Seasons & 4 & 0.06 & $10.79 *$ & 0.73 & 2.55 & 0.42 & 2.24 & 0.97 & 2.06 & $6.79 * *$ \\
\hline Genotypes & 10 & $7.65^{* *}$ & $17.31 * *$ & $5.26 * *$ & $16.15^{* *}$ & 0.86 & $8.33^{* *}$ & $5.50^{*}$ & $6.32 * *$ & $5.39 * *$ \\
\hline Durum vs Bread & 1 & $16.57 * *$ & $48.09 * *$ & $20.91 * *$ & $42.68^{* *}$ & 0.16 & $28.91 * *$ & 2.92 & 3.46 & $5.58^{*}$ \\
\hline Among durum & 7 & $8.37 * *$ & $15.57 * *$ & $3.70^{* *}$ & $13.67 * *$ & 0.76 & $6.94 * *$ & $6.24 * *$ & $8.46^{* *}$ & $5.13 * *$ \\
\hline Among Bread & 2 & 0.67 & 8.00 & 2.89 & $11.56^{*}$ & 1.56 & 2.89 & 4.22 & 0.22 & $6.22 *$ \\
\hline $\mathbf{S} \times \mathbf{G}$ & 10 & $4.28 *$ & 6.40 & $3.39 * *$ & $10.86^{* *}$ & 0.81 & 1.61 & 3.20 & $5.44 * *$ & $2.53 * *$ \\
\hline Error & 40 & 1.93 & 3.45 & 1.13 & 2.81 & 1.09 & 1.24 & 1.77 & 1.06 & 0.85 \\
\hline \multirow[t]{2}{*}{ Heritability (B.S.) } & - & 0.17 & 0.29 & 0.14 & 0.14 & 0.01 & 0.45 & 0.15 & 0.05 & 0.25 \\
\hline & & \multicolumn{9}{|c|}{ Mean squares } \\
\hline S. O. V. & df & FLW & FLL & Ped. L & PLHT & Out & Pub. & $\begin{array}{l}\text { Ear } \\
\text { Den }\end{array}$ & $\begin{array}{c}\text { Ear } \\
\text { Orint }\end{array}$ & Ear Sh \\
\hline Seasons & 1 & 6.06 & 3.88 & 2.18 & 0.97 & & & $19.64 * *$ & 3.88 & 4.91 \\
\hline R./Seasons & 4 & 3.15 & 3.88 & 1.21 & 0.61 & & & $3.09 *$ & 2.61 & 2.91 \\
\hline Genotypes & 10 & $10.33^{* *}$ & $12.99 * *$ & $8.00 * *$ & $2.15^{* *}$ & & & $8.86^{* *}$ & $4.97 *$ & $9.37 * *$ \\
\hline Durum vs Bread & 1 & $16.16^{* *}$ & 0.16 & $30.56^{* *}$ & $15.76^{* *}$ & & & $57.58^{* *}$ & 0.01 & 5.11 \\
\hline Among Durum & 7 & $12.19 * *$ & $13.71 * *$ & $6.05^{* *}$ & 0.37 & & & $2.46^{*}$ & $4.75^{*}$ & $5.80 * *$ \\
\hline Among Bread & 2 & 0.89 & $16.89^{* *}$ & 3.56 & 1.56 & & & $6.89^{* * *}$ & $8.22 *$ & $24.00^{* *}$ \\
\hline $\mathbf{S} \times \mathbf{G}$ & 10 & 0.73 & 1.75 & $6.45^{* *}$ & 0.84 & & & 1.64 & 2.55 & 1.18 \\
\hline Error & 40 & 1.82 & 2.28 & 1.48 & 0.67 & & & 0.96 & 1.74 & 1.31 \\
\hline Heritability (B.S.) & - & 0.52 & 0.47 & 0.08 & 0.23 & & & 0.5 & 0.17 & 0.52 \\
\hline
\end{tabular}

*, ** Significant differences at $\mathrm{P}<0.05$ and $\mathrm{P}<0.01$, respectively.

\subsection{Correlation coefficients among traits}

Correlations analysis among the studied traits

(Table 6) showed that Ear Den was positively correlated with FCL $(\mathrm{r}=0.51, P<0.01)$, WSH $(\mathrm{r}=0.57$, $P<0.01)$, W. Ped $(\mathrm{r}=0.60, P<0.01)$ and Ped. L $(\mathrm{r}=0.53$, $P<0.01)$. However, a negative correlation was found between Ear Den and PLHT $(\mathrm{r}=-0.52, P<0.01)$. Significant and positive correlation was found between GH and CLAR ( $\mathrm{r}=0.43, P<0.05)$, whereas significant and negative correlation was obtained between $\mathrm{GH}$ and FLL $(\mathrm{r}=-0.44, \quad P<0.05)$. Meantime, WSH was positively correlated with $\mathrm{W}$. Ear $(\mathrm{r}=0.53, P<0.01), \mathrm{W}$. Ped ( $\mathrm{r}=0.56, P<0.01)$, and Ped. L $(\mathrm{r}=0.43, P<0.05)$, but, it was negatively correlated with PLHT (-0.53,
$P<0.01)$. Positive and highly significant correlations were found between FCL with WSH $(\mathrm{r}=0.55, \mathrm{P}<0.01)$ and Ped. $\mathrm{L}(\mathrm{r}=0.54, \mathrm{P}<0.01)$. Similarly, positive correlations were found between HRAR with FLAT ( $\mathrm{r}=$ $0.41, \mathrm{P}<0.05)$ and PLHT $(\mathrm{r}=0.68, \mathrm{P}<0.01)$.

\subsection{SSR markers data analysis}

Out of 21 SSR primer pairs used for screening eight durum and three bread wheat genotypes, a total number of 115 bands were generated, which ranged from 1 band for Xgwm165-4D, Xgwm437-7D and Xgwm325-6D to 13 bands for Xgwm155-3A, with an average of 5.48 bands per primer. Of 115 bands generated, 97 bands were polymorphic with an average of 4.62 bands per primer. The lowest 


\begin{tabular}{|c|c|c|c|c|c|c|c|c|c|c|c|c|c|c|c|c|c|}
\hline Traits & 0 & 已 & $\underline{\underline{z}}$ & $\underset{\Xi}{\mho}$ & $\underset{ت}{ت}$ & $\frac{7}{5}$ & $\sum$ & $\stackrel{1}{\ddot{1}}$ & $\begin{array}{l}2 \\
2 \\
2\end{array}$ & 3 & $\vec{a}$ & تح & $\underline{\underline{a}}$ & 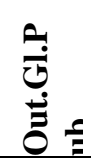 & 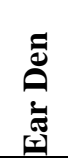 & 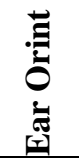 & 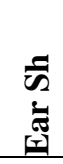 \\
\hline GH & 1.00 & & & & & & & & & & & & & & & & \\
\hline FCL & 0.11 & 1.00 & & & & & & & & & & & & & & & \\
\hline HRAR & -0.13 & -0.27 & 1.00 & & & & & & & & & & & & & & \\
\hline CLAR & $0.43^{*}$ & -0.02 & -0.04 & 1.00 & & & & & & & & & & & & & \\
\hline FLAT & 0.06 & 0.14 & $0.41 *$ & -0.29 & 1.00 & & & & & & & & & & & & \\
\hline WSH & 0.21 & $0.55^{* *}$ & -0.33 & $0.45^{*}$ & $\begin{array}{c}- \\
0.13\end{array}$ & 1.00 & & & & & & & & & & & \\
\hline WBL & -0.02 & 0.02 & 0.06 & 0.31 & 0.24 & 0.35 & 1.00 & & & & & & & & & & \\
\hline W. Ear & 0.01 & 0.40 & -0.08 & 0.35 & 0.01 & $0.53 * *$ & $0.54 * *$ & 1.00 & & & & & & & & & \\
\hline W. Ped & 0.03 & 0.36 & -0.04 & 0.13 & $\begin{array}{c}- \\
0.11\end{array}$ & $0.56^{* *}$ & -0.01 & 0.35 & 1.00 & & & & & & & & \\
\hline FLW & -0.19 & 0.19 & -0.11 & 0.04 & $\overline{-}-$ & 0.16 & -0.04 & 0.01 & -0.11 & 1.00 & & & & & & & \\
\hline FLL & ${ }^{-} \overline{4} 4^{*}$ & -0.21 & -0.39 & -0.17 & $\begin{array}{c}- \\
0.30\end{array}$ & 0.09 & 0.00 & 0.03 & -0.03 & 0.39 & 1.00 & & & & & & \\
\hline Ped. L & 0.23 & $0.54 * *$ & -0.18 & 0.20 & 0.10 & $0.43^{*}$ & -0.24 & 0.02 & $0.41^{*}$ & 0.02 & $\begin{array}{c}- \\
0.14\end{array}$ & 1.00 & & & & & \\
\hline PLHT & -0.31 & -0.25 & $0.68 * *$ & -0.21 & 0.34 & $0.53^{-}$ & 0.06 & 0.04 & -0.25 & 0.17 & $\begin{array}{c}- \\
0.19\end{array}$ & -0.32 & 1.00 & & & & \\
\hline Out.Gl.Pub & 0.01 & 0.00 & -0.27 & -0.22 & $\overline{-}$ & -0.18 & -0.17 & $\overline{-}$ & -0.32 & 0.20 & 0.06 & -0.11 & -0.10 & 1.00 & & & \\
\hline Ear Den & 0.18 & $0.51 * *$ & -0.30 & 0.40 & $\overline{-}$ & $0.57 * *$ & 0.01 & 0.36 & $0.60 * *$ & -0.25 & $\overline{0} \overline{25}$ & $0.53 * *$ & $\overline{-}^{-}$ & $\overline{-}$ & 1.00 & & \\
\hline Ear Orint & -0.25 & 0.24 & -0.21 & -0.09 & $\overline{-}$ & 0.40 & 0.36 & 0.17 & 0.05 & $0.47 * *$ & 0.36 & -0.07 & -0.12 & $0 . \overline{27}$ & $\overline{-}-$ & 1.00 & \\
\hline Ear Sh & 0.15 & 0.00 & -0.14 & 0.11 & $\begin{array}{c}- \\
0.17 \\
\end{array}$ & 0.31 & -0.11 & $\begin{array}{c}- \\
0.15 \\
\end{array}$ & $0.47 * *$ & -0.24 & $\begin{array}{c}- \\
0.13 \\
\end{array}$ & 0.30 & -0.29 & $\begin{array}{c}- \\
0.09 \\
\end{array}$ & 0.37 & $\begin{array}{c}- \\
0.17 \\
\end{array}$ & 1.00 \\
\hline
\end{tabular}

*, ** Significant differences at $\mathrm{P}<0.05$ and $\mathrm{P}<0.01$, respectively.

polymorphism $(0 \%)$ was observed with Xgwm33-1A and Xgwm325-6D, whereas the highest polymorphism (100\%) was produced by ten SSRs, with $84.35 \%$ averaged polymorphism. The polymorphism information content (PIC) values ranged from 0 for Xgwm33-1A and Xgwm325-6D to 0.48 for Xgwm160$4 \mathrm{~A}$, with an average of 0.26 . The highest MI value (3.96) was obtained for Xgwm95 and the lowest MI value (0.0) was observed in Xgwm33-1A and Xgwm325-6D (Table 7).

Among seven SSR primer pairs represented the A genome, a total number of 61 bands were generated, with an average of 8.71 bands per primer. A high polymorphism (81.97\%) was found for the A genome with 50 polymorphic bands with an average of 7.14 per primer. In addition, out of seven SSR primer pairs represented the B genome, a total of 32 DNA bands were detected, with an average of 4.57 per primer. A total of 29 polymorphic bands were obtained $(90.63 \%$ polymorphism), with an average of 4.14 per primer. Otherwise, out of seven SSR primer pairs represented the D genome, 22 bands were generated, with an average of 3.14 per primer. Of which, 18 polymorphic bands were obtained ( $81.82 \%$ polymorphism), with an average of 2.57 per primer. 
Mahmoud A. El-Rawy., 2020

Table 7. Number of total bands, polymorphic bands, PIC, MI and RP for each SSR primer.

\begin{tabular}{ccccccc}
\hline Primer & TAB & NPB & POL & PIC & MI & RP \\
\hline Xgwm33-1A & 3.00 & 0.00 & 00.00 & 0.00 & 0.00 & 0.00 \\
Xgwm95-2A & 11.00 & 11.00 & 100.00 & 0.36 & 3.96 & 6.00 \\
Xgwm155-3A & 13.00 & 7.00 & 53.84 & 0.39 & 2.73 & 6.43 \\
Xgwm160-4A & 2.00 & 2.00 & 100.00 & 0.48 & 0.96 & 2.63 \\
Xgwm186-5A & 11.00 & 11.00 & 100.00 & 0.21 & 2.31 & 4.30 \\
Xgwm459-6A & 10.00 & 9.00 & 90.00 & 0.28 & 2.52 & 4.18 \\
Xgwm63-7A & 11.00 & 10.00 & 90.09 & 0.34 & 3.40 & 8.00 \\
\hline total & 61 & 50 & 81.97 & - & - & - \\
\hline Xgwm18-1B & 2.00 & 2.00 & 100.00 & 0.28 & 0.56 & 3.27 \\
Xgwm111-2B & 2.00 & 1.00 & 50.00 & 0.17 & 0.17 & 1.81 \\
Xgwm389-3B & 4.00 & 4.00 & 100.00 & 0.26 & 1.04 & 1.24 \\
Xgwm513-4B & 5.00 & 5.00 & 100.00 & 0.31 & 1.55 & 1.64 \\
Xgwm408-5B & 6.00 & 5.00 & 83.33 & 0.38 & 1.90 & 6.90 \\
Xgwm626-6B & 7.00 & 7.00 & 100.00 & 0.26 & 1.82 & 4.00 \\
Xgwm577-7B & 6.00 & 5.00 & 83.33 & 0.19 & 0.95 & 3.36 \\
\hline total & 32 & 29 & 90.63 & - & - & - \\
\hline Xgwm458-1D & 3.00 & 2.00 & 66.67 & 0.40 & 0.80 & 1.27 \\
Xgwm261-2D & 2.00 & 1.00 & 50.00 & 0.17 & 0.17 & 0.18 \\
Xgwm3-3D & 4.00 & 3.00 & 75.00 & 0.40 & 1.20 & 3.45 \\
Xgwm165-4D & 1.00 & 1.00 & 100.00 & 0.17 & 0.17 & 3.45 \\
Xgwm190-5D & 10.00 & 10.00 & 100.00 & 0.26 & 2.60 & 3.64 \\
Xgwm325-6D & 1.00 & 0.00 & 00.00 & 0.00 & 0.00 & 0.00 \\
Xgwm437-7D & 1.00 & 1.00 & 100.00 & 0.30 & 0.30 & 0.36 \\
\hline total & 22 & 18 & 81.81 & - & - & - \\
\hline Grand total & 115 & 97 & 84.35 & - & - & - \\
\hline Average & $\mathbf{5 . 4 8}$ & $\mathbf{4 . 6 2}$ & $\mathbf{7 8 . 5 7}$ & $\mathbf{0 . 2 6 1}$ & $\mathbf{1 . 4 1}$ & $\mathbf{3 . 2 5}$ \\
\hline
\end{tabular}

Total amplified bands (TAB), No. of Polymorphic bands (NPB), \% of Polymorphism (POL)

Polymorphic information Content (PIC), Marker index (MI), Resolving power (RP)

Based on single marker analysis and among 21 SSR markers analysed, 11 were significantly associated with phenotypic traits (Table 8). Three markers (Xgwm181B, Xgwm459-6B and Xgwm160-4A) were associated with Ear Sh, with $\mathrm{R}^{2}$ values of $38.81,51.29$ and $37.26 \%$, respectively. Two markers (Xgwm437-7D and Xgwm577-7B) were associated with PLHT $\left(\mathrm{R}^{2}=79.11\right.$ and $37.68 \%$, respectively). Six markers, each of which was associated with a single trait, i.e. Xgwm408-5B with HRAR, Xgwm389-3B with FLW, Xgwm111-2B with W. Ped, Xgwm190-5A with FCL, Xgwm95-2A with Ear Den and Xgwm186-5D with CLAR, with $\mathrm{R}^{2}$ values of 46.23, 82.79, 58.09, 62.82, 63.77 and 53.39 $\%$, respectively (Fig 1).

SSR markers analysis showed that four SSR markers generated four specific bands which were able to distinguish durum from bread wheat genotypes. A specific band generated by Xgwm155-3A (120 bp) was detected only in durum wheat genotypes, while three bands generated by Xgwm513-4B (128 bp),
Xgwm458-1D (121 bp) and Xgwm186-5A (298 bp) were present in bread wheat but absent in durum wheat genotypes. Two unique bands generated by Xgwm4085B and Xgwm458-1D (365 and $232 \mathrm{bp)}$ were present in all durum and bread wheat genotypes but, it was absent only in the durum G1, G2, G3, G4 and G5 Bani Sueif wheat genotype. Two unique bands generated by Xgwm95-2A and Xgwm437-7D (431 and 105 bp) were present in G9 and G10 of bread wheat genotypes but, it was absent in the all durum and bread wheat genotypes. A single specific band generated with Xgwm63-7A primer $(510 \mathrm{pb})$ was present only in G6, G7 and G8 (Sohag) of durum wheat genotypes (Table 9).

The dendrogram constructed based on phenotypic distance matrix obtained using morphological traits showed that all studied cultivars were divided into two main clusters. The first main 
Scientific Journal of Agricultural Sciences 2 (1): 00-00, 2020

Table 8. SSR markers associated with morphological characters based on single marker regression.

\begin{tabular}{llccc}
\hline Trait & Marker & Specific band (bp) & P value & $\mathbf{R}^{2}$ \\
\hline \multirow{2}{*}{ Ear Sh } & Xgwm160-4A & 117 & 0.046 & 37.26 \\
& Xgwm459-6A & 265 & 0.013 & 51.29 \\
\hline HRAR & Xgwm18-1B & 350 & 0.041 & 38.81 \\
\hline FLW & Xgwm408-5B & 111 & 0.021 & 46.23 \\
\hline W. Ped & Xgwm111-2B & 122 & 0.001 & 82.79 \\
\hline FCL & Xgwm190-5D & 117 & 0.006 & 58.09 \\
\hline Ear Den. & Xgwm95-2A & 210 & 0.004 & 62.82 \\
\hline \multirow{2}{*}{ PLHT } & Xgwm577-7B & 163 & 0.047 & 63.77 \\
\hline CLAR & Xgwm437-7D & 944 & 0.044 & 37.68 \\
\hline
\end{tabular}

R2, (the coefficient of determination) indicates the percentage of phenotypic variation explained by the marker.

Table 9. Unique DNA bands generated by some SSR markers.

\begin{tabular}{|c|c|c|}
\hline Genotypes & Positive & Negative \\
\hline Durum wheat & Xgwm155-3A (120 bp) & $\begin{array}{l}\text { Xgwm513-4B (128 bp), Xgwm458-1D } \\
\text { (121 bp) Xgwm186-5A (298 bp) }\end{array}$ \\
\hline Bread & $\begin{array}{c}\text { Xgwm513-4B (128 bp), Xgwm458-1D (121 } \\
\text { bp) Xgwm186-5A (298 bp) }\end{array}$ & Xgwm155-3A (120 bp) \\
\hline $\begin{array}{l}\mathrm{G} 1, \mathrm{G} 2, \mathrm{G} 3, \mathrm{G} 4 \text { and } \mathrm{G} 5 \\
\text { (Bani Sueif) }\end{array}$ & - & $\begin{array}{l}\text { Xgwm408-5B (365bp) } \\
\text { Xgwm458-1D (232bp) }\end{array}$ \\
\hline G6, G7 and G8 (Sohag) & Xgwm63-7A(510pb) & - \\
\hline Gemmiza & $\begin{array}{l}\text { Xgwm95-7D(431bp) } \\
\text { Xgwm437-2A(105bp) }\end{array}$ & - \\
\hline
\end{tabular}

cluster contained all durum wheat genotypes except G1 and the seconded main cluster were contained all bread wheat genotypes with G1 (Fig.2). Meanwhile, the dendrogram constructed based on similarity matrix obtained by SSR markers showed that the studied cultivars were grouped into two main clusters. The first main cluster contained G6 and G7 with all bread wheat genotypes and the seconded main cluster contained G8 with G1, G2, G3, G4 and G5 (Bani Suif) genotypes, which all belong to durum wheat. A significant and positive correlation $(r=0.25, p<0.05)$ was found between the matrices obtained by phenotypic data and molecular SSR markers (Fig. 3). 
Mahmoud A. El-Rawy., 2020

Xgwm 408

Xgwm 459
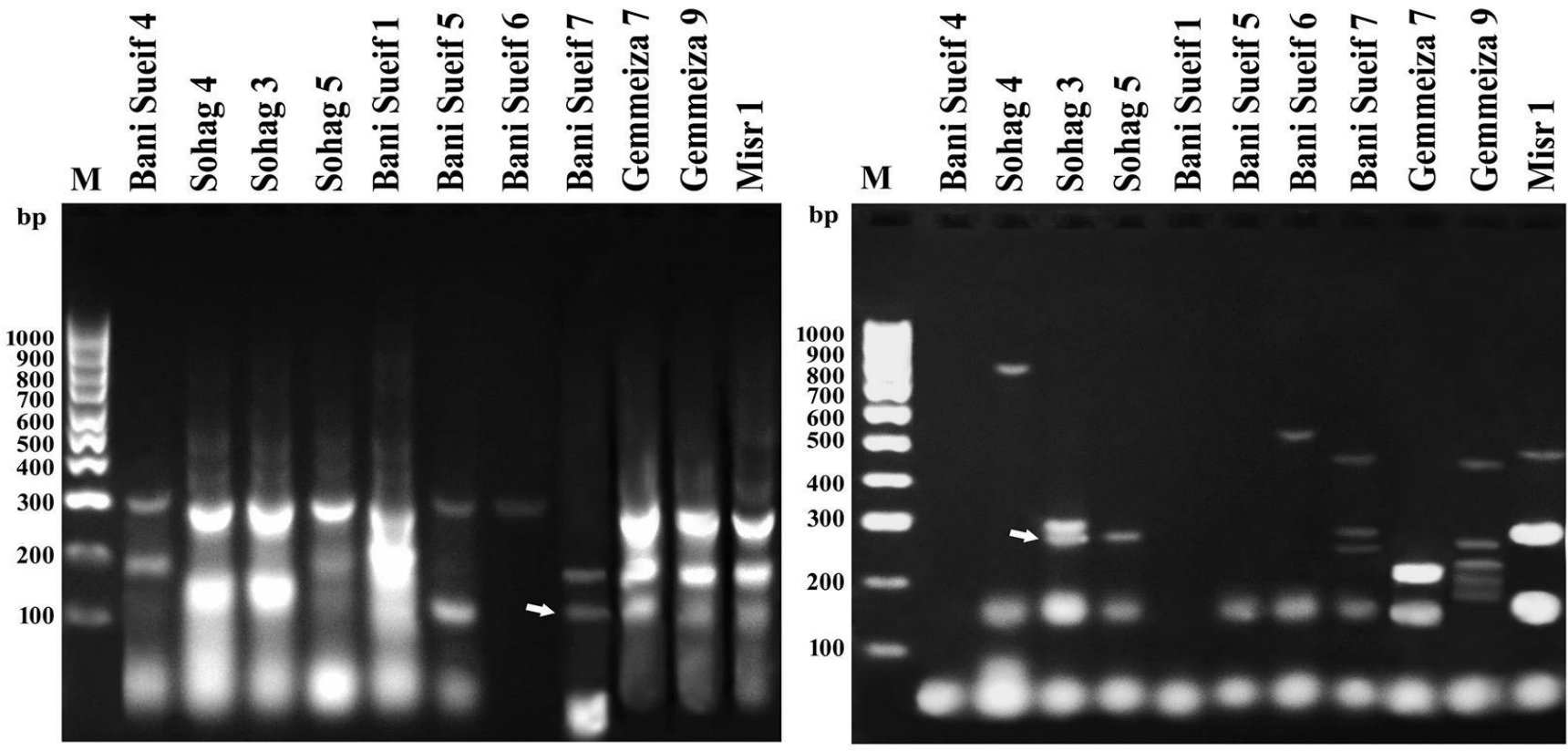

Xgwm 190

Xgwm 186

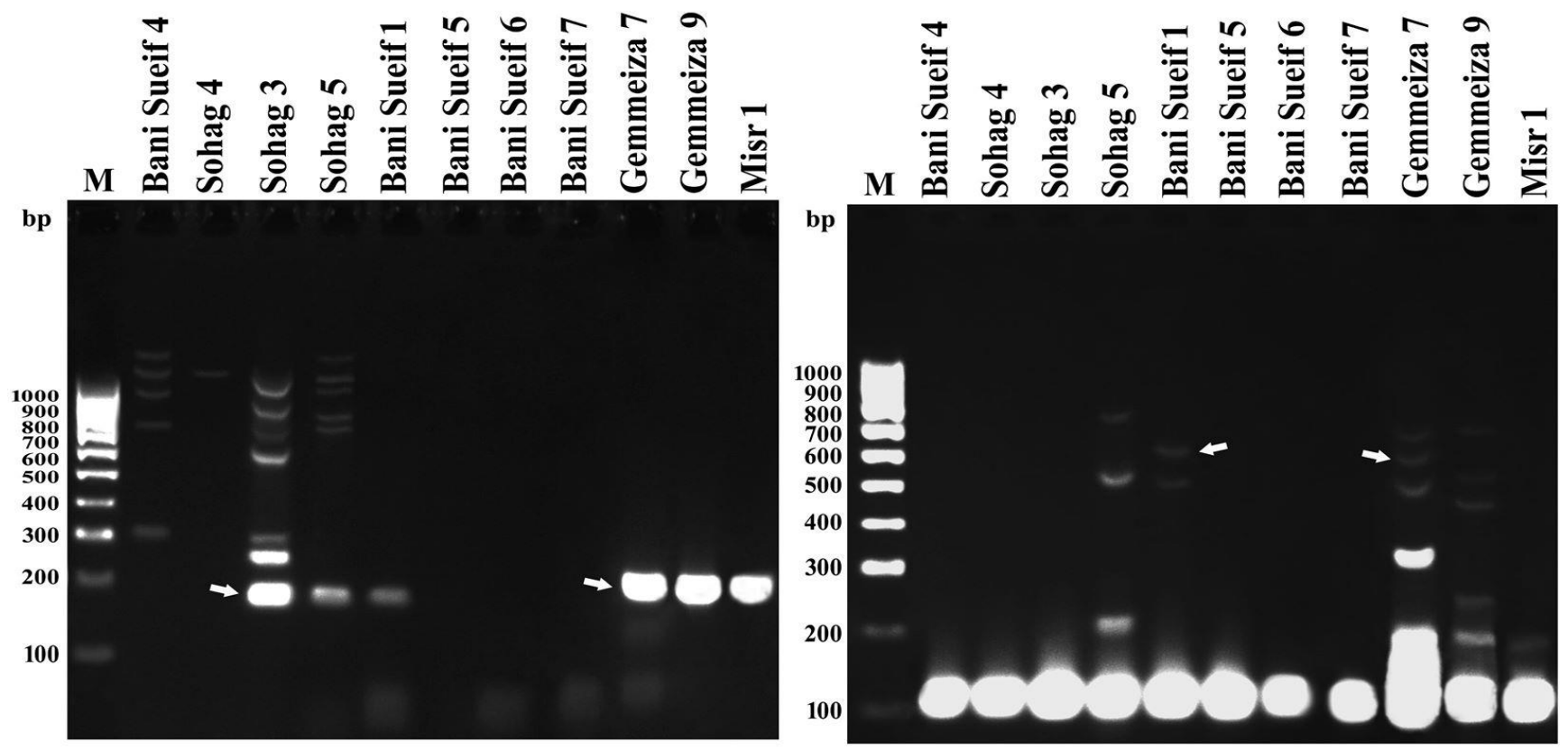

Fig 1. PCR amplification patterns obtained using Xgwm 408, Xgwm 459, Xgwm 190 and Xgwm 186 markers in the studied cultivars. M: A 100bp DNA ladder 

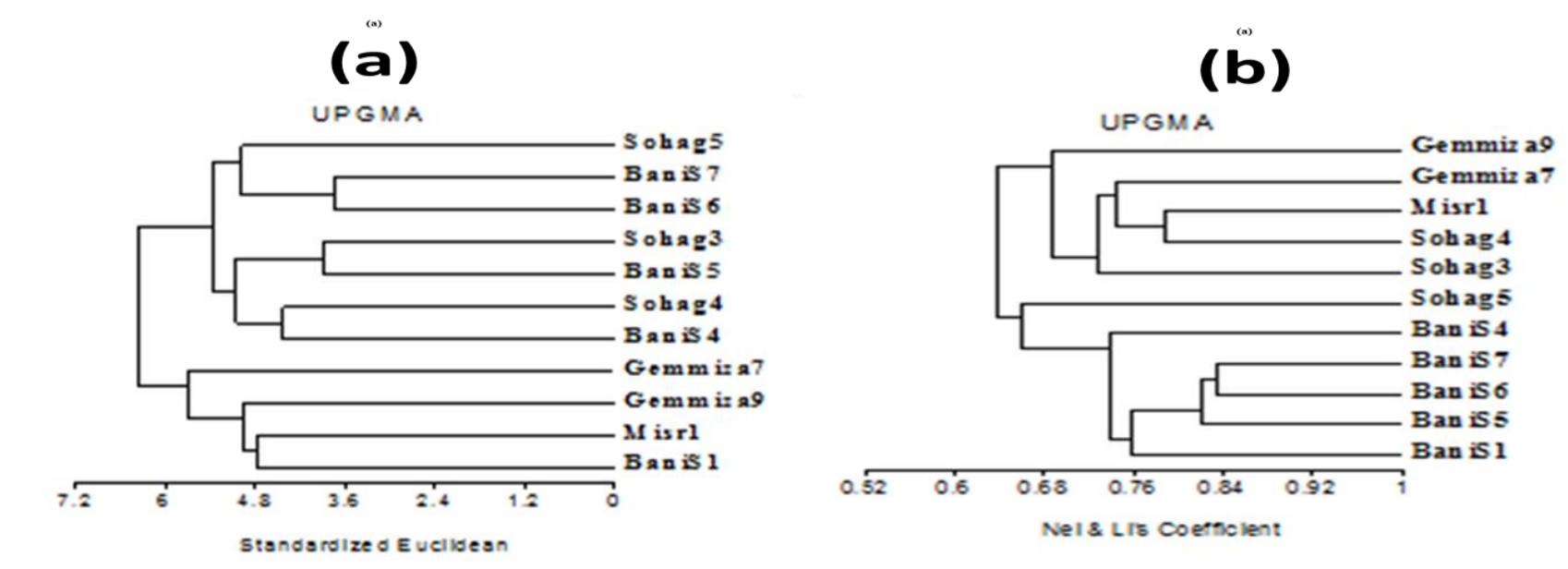

Fig 2. Dendrogram of genetic similarities using SSR data (a), Dendrogram of genetic distance using morphological data (b).

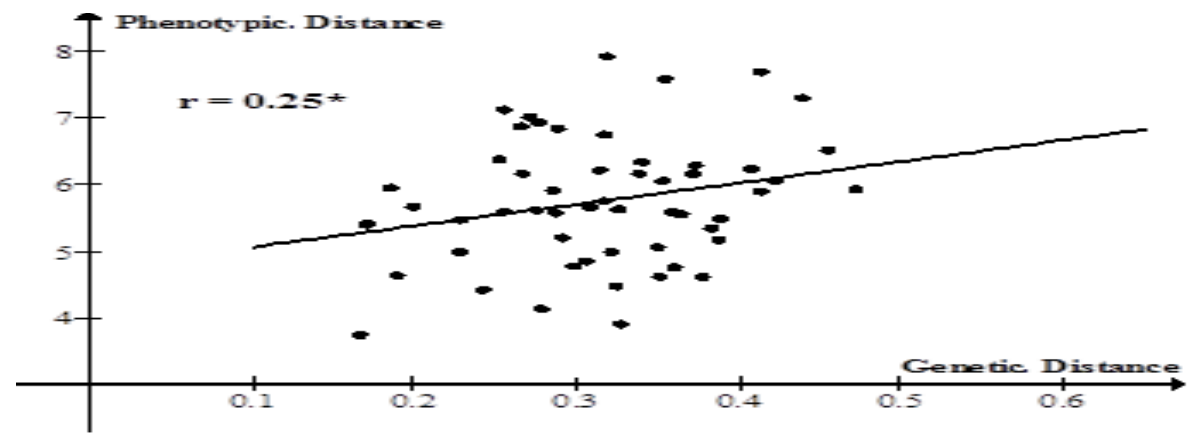

Fig 3. Correlation between genetic and phenotypic distance.

\section{Discussion}

The present study was carried out to assess the genetic diversity among eight durum and three bread wheat genotypes based on 17 morphological traits and 21 SSR markers. Genetic variation between the local durum and bread wheat cultivars was found in most of the studied morpho-phosiological traits and molecular markers. Significant and highly significant differences were obtained among the genotypes for all the traits except FLAT and Out.Gl.Pub. Highly significant differences were obtained between durum and bread wheat genotypes for GH, FCL, HRAR, CLAR, WSH, FLW, Ped. L, PLHT and Ear Den, while nonsignificant differences were observed for FLAT, WBL, W. Ear, FLL, Out.Gl.Pub, Ear Orint and Ear Sh. These agro-morphological features could be useful in exploring and selecting plant material for breeding purposes (Malik et al., 2014). Hassan et al. (2016) reported that the grain yield was higher in durum wheat than bread wheat while the plant height was higher in bread wheat.

Interestingly in the present study, mean values of all wax traits studied in durum wheat were greater in magnitude than those of bread wheat. In this regard, Clarke et al. (1993) found an association between visual ratings of glaucousness and epicuticular wax quantity in durum and bread wheat genotypes, and they found that the wax quantity was $44 \%$ greater for glaucous than those for nonglaucous durum genotypes, and $32 \%$ greater for glaucous than for nonglaucous bread wheat genotypes. They also found that wax quantity of the flag leaf blade, sheath, and spike was greater for durum than for bread wheat genotypes. Willick et al. (2018) observed that greater wax crystal density on the adaxial and abaxial surfaces of the drought-tolerant flag leaves corresponded to higher harvest indices.

Moderate broad-sense heritability estimates were obtained for WSH (0.45), FLW (0.52), FLL 


\section{Mahmoud A. El-Rawy., 2020}

(0.47), Ear Den (0.50) and Ear Sh (0.52), whereas low heritability was found for GH (0.17), FCL (0.29), HRAR (0.14), CLAR (0.14), WBL (0.15), W. Ped (0.25), PLHT (0.23) and Ear Orint (0.17). Moderate to high heritability were recorded for Spike Length $(46.03 \%)$, flag leaf area $(41.39 \%)$, 1000-grain weight $(58.58 \%)$, relative water contents $(58.89 \%)$ (Ijaz and Smiullah 2013). High heritability and genetic advance estimates were reported for flag leaf area (Saleem et al. 2016).

Positive and highly significant correlations were observed between WSH with W. Ear and W. Ped also, between W. Ear and WBL. Positive and highly significant correlations found obtained between FCL with WSH, Ped. L and Ear Den. Similarly, positive and significant correlations were found between HRAR with FLAT and PLHT. These results were in agreement with Feltaous (2019) where all wax characteristics studied in different parts of the plant were positively correlated each other. Significant positive correlations were observed between peduncle wax state with leafblade wax, leaf-sheath wax and ear wax by Malik et al. (2013). Glaucousness is one of the most eye-catching traits and has been long used as a morphological marker in wheat genetic studies for $\sim 80$ years. Its adaptive value in improving crop tolerance to drought and heat was recognized in 1980s (Tsunewaki et al. 1999).

Molecular marker analysis showed that the polymorphism obtained using 21 SSR markers ranged from 0 to 100 with an average of $84.35 \%$. The PIC values ranged from 0 to 0.48 with an average of 0.26 per marker. The highest MI value (3.96) was obtained by the Xgwm95. Prasad et al. (2000) reported that one to 13 alleles per locus in 55 wheat genotypes with 20 SSR markers, with a PIC value ranging from 0.21 to 0.90 , with an of average of 0.68. Mardi et al. (2011) indicated that 2 to 10 alleles per locus using 19 SSR markers were found with 122 durum wheat genotypes. These results supported that SSR markers are effective for estimating the genetic diversity as previously reported by several investigations (Hassan 2016).

In the present study, although, the A genome contained the highest mean number of alleles ( 8.71 per marker) followed by the B genome (4.57 per marker) however, a high polymorphism was found only that in the B genome $(90.63 \%)$ followed by the A genome $(81.97 \%)$. In accordance, Chao et al. (2007) reported that a high polymorphism among wheat genotypes in the $\mathrm{B}$ genome followed by $\mathrm{D}$ and $\mathrm{A}$ genomes. In addition, microsatellite distribution was abundance on chromosomes of the B genome followed by chromosomes of the A and D genomes (Jaiswal et al. 2017). Chen and $\mathrm{Li}$ (2007) found that the ranking of average locus diversity per genome was $\mathrm{D}>\mathrm{B}>\mathrm{A}$ in synthetic hexaploid wheat genotypes also, they reported that the $\mathrm{D}$ genome contained the highest mean number of alleles (6.32) followed by A and B genomes (6.13 and 5.94, respectively). The highest mean PIC was recorded in the A genome (0.7540), followed by the $\mathrm{D}$ genome (0.7482) and the B genome $(0.7361)$ as reported by Wang et al. (2013). In the present study, low number of alleles for the D genome was found due to the low number of used genotypes containing the $\mathrm{D}$ genome.

Single marker analysis showed that 11 SSR markers were significantly associated with phenotypic traits. The markers Xgwm18-1B, Xgwm459-6B and Xgwm160-4A, were associated with Ear shape, Xgwm95-2A was associated with Ear Density, and Xgwm437-7D and Xgwm577-7B were found to be associated with PLHT. Sheoran et al. (2019) found strong co-localized loci for glume pubescence, spike length, plant height, and awn color located on chromosome 1B in wheat. Wei et al. (2010) identified a few stable plant height QTLs on chromosomes 2A, 2B, 2D, 3B, 4B, 5A, 5D, 7B, and 7D. A QTL located on chromosome $3 \mathrm{~B}$ was associated with increased biomass, grain number and grain weight following heat stress in bread wheat (Thomelin et al. 2019). Five SSR markers, each of which was associated with a single trait, i.e. Xgwm408-5B with HRAR, Xgwm389-3B with FLW, Xgwm190-5A with FCL, Xgwm95-2A with Ear. Dens and Xgwm186-5D with Auricles CLAR. Keller et al. (1999) identified eight QTLs for flag leaf width on chromosomes 1A, 1B, $2 \mathrm{~A}, 3 \mathrm{~B}, 5 \mathrm{~A}, 5 \mathrm{~B}$ and $6 \mathrm{~A}$, which account for $59.5 \%$ of the phenotypic variations. In durum wheat, QTLs for flag leaf angle, length, and width were mapped to chromosomes 2A, 3B, 5B, 7A, and 2B by Isidro et al. (2012). In addition, Fan et al. (2015) identified 38 additive QTLs for flag leaf width, flag leaf length and flag leaf area on 12 wheat chromosomes, explaining $3.96-27.68 \%$ of the phenotypic variations. Liu et al. (2018) found 23 putative QTLs for flag leaf length, width, area, and flag leaf angle on chromosomes $1 \mathrm{~B}$, 2B, 3A, 3D, 4B, 5A, 6B, 7B, and 7D. Twenty stable QTLs were identified for flag leaf morphology and could be potentially useful for genetic improvement of drought tolerance in wheat through QTL pyramiding (Yang et al. 2016).

In the present study, some of the most important traits in wheat were studied, namely ear waxiness, waxiness of flag leaf sheath, leaf blade waxiness and waxiness of peduncle. In this regard, it has been reported that the wheat leaf, stem and, in some cases, spike surfaces are coated with cuticular waxes, 
which confers a glaucousness characteristic (Jensen and Driscoll 1962). The outermost wax layer functions as a barrier between plants and their environment, in defending plants against the biotic and abiotic stresses, such as drought, phytophagous insects, pathogens, solar radiation, and freezing temperatures (Jenks and Ashworth 1999). The wax on wheat leaves and stems is mainly controlled by two sets of genes: glaucousness loci (W1 and W2) and non-glaucousness loci (Iw1 and Iw2) (Ping et al. 2015). The wax production genes W1 and W2 contribute while Iw1 and Iw2 inhibit the glaucousness (Bi et al. 2017). In the present study, the SSR marker Xgwm111-2B was found to be associated with waxiness of peduncle with $\mathrm{R}^{2}$ value of $58.09 \%$. $\mathrm{Wu}$ et al. (2013) reported that the $\mathrm{W} 1$ gene was located on the chromosome arm 2BS between markers Xgwm210 and Xbarc35, Eleven Iw1 and eight Iw2 linked expressed sequence tag (EST) markers were developed and mapped on the distal regions of chromosomes 2BS and 2DS, respectively.

In the present study, a significant with low correlation $(r=0.25)$ was found between the dissimilarity matrix generated from the phenotypic data and that obtained from the molecular data, indicating that the SSR markers were able to bind to effective regions in the genome. However, the SSR markers did not adequately sample the genomic regions that were relevant for the phenotypic differentiation of the studied cultivars. Dendrogram constructed based on similarity matrix obtained from SSR markers, the studied cultivars were clustered into two main clusters. The first main cluster contained all bread wheat genotypes and the seconded main cluster contained most of the durum wheat genotypes. The same result was obtained from dendrogram constructed based on phenotypic data. These finding supported that SSR markers and morphological traits were found to be useful for the assessment of genetic diversity in wheat. Benin et al. (2012) found significant correlations, ranged from low (0.45) to moderate $(0.67)$, between the distance measures based on AFLP markers and hybrid performance in spring wheat. Al-Ashkar et al. (2020) found a significant correlation between the morphological and genetic distances $(r=0.51, p<$ 0.0001 ) in wheat under salinity stress conditions. The cluster analysis based on SSR markers showed correlation with the grouping of particular genotypes based on agro-morphological characters (Zarkti et al. 2010), suggesting that the characterization based on agro-morphological traits and SSR markers will be a useful tool to the breeders to choose genotypes with appropriate. Very weak correlations between morphologic and molecular data were also reported by
Cupic et al. (2009). No correlations between phenotypic and molecular data were found Petrovic et al. (2017), implying that both types of data should be used for genetic diversity estimates in order to cover wider variability between tested cultivars.

In conclusion, the phenotypic data and molecular markers were effective in estimating the genetic variability between wheat cultivars. The study indicated the presence of abundant genetic variability among some of the important Egyptian cultivars. Significant positive correlation found between the phenotypic and genotypic distance indicated that SSR markers were able to bind to effective regions in the genome. Single marker analysis revealed that eleven markers were associated with phenotypic traits which can be useful for markers-assisted breeding in the tested wheat genotypes. However, additional markers analyses are still required to validate their effectiveness in wheat breeding programs.

\section{REFERENCES}

Aghaee M, Mohammadi R, Nabovati (2010). Agromorphological characterization of durum wheat accessions using pattern analysis. Aust. J. Crop Sci. 4:505-514.

Al-Ashkar I, Alderfasi A, Romdhane WB, Seleiman MF, El-Said RA, Al-Doss A. (2020). Morphological and Genetic Diversity within Salt Tolerance Detection in Eighteen Wheat Genotypes. Plants 9(3): 287. DOI: 10.3390/plants9030287

Arain SM, Sial MA, Jamali KD, Laghari KA. (2018). Grain yield performance, correlation, and cluster analysis in elite bread wheat (Triticum aestivum L.) lines. Acta Agrobot. 71(4):1747.

Benin G, Matei G, Costa de Oliveira A, Silva GO, Hagemann TR, Lemes da Silva C, Pagliosa ES, Beche E. (2012). Relationships between four measures of genetic distance and breeding behavior in spring wheat. Genet. Mol. Res. 11(3):2390-2400.

Bi H, Kovalchuk N, Langridge P, Tricker PJ, Lopato S, Borisjuk N. (2017). The impact of drought on wheat leaf cuticle properties. BMC Plant Biol. 17: 85

Chao SM, Zhang WJ, Dubcovsky J, Sorrells M. (2007). Evaluation of genetic diversity and genomewide linkage disequilibrium among U.S. wheat (Triticum aestivum L.) germplasm representing different market classes. Crop Sci. 47(3):1018-1030.

Chen GY, Li LH. (2007). Detection of genetic diversity in synthetic hexaploid wheats using microsatellite markers. Agric. Sci. China 6(12):14031410 . 


\section{Mahmoud A. El-Rawy., 2020}

Clarke FM, McCaig TN, DePauw RM. (1993). Relationship of glaucousness and epicuticular wax qrnntity of wheat. Can. J. Plant Sci. 73: 961-967.

Cupic T, Tucak M, Popovics S, Bolaric S, Grljusic S, Kozumplik V. (2009). Genetic diversity of pea (Pisum sativum L.) genotypes assessed by pedigree, morphological and molecular data. J. Food Agric. Environ. 7(3-4): 343- 348.

EI-Rawy MA, Hassan MI. (2014). Effectiveness of drought tolerance indices to identify tolerant genotypes in bread wheat (Triticum aestivum L.). J. Crop Sci. Biotech. 17 (4): 255-266.

El-Rawy MA, Youssef M. (2014). Evaluation of drought and heat tolerance in wheat based on seedling traits and molecular analysis. J. Crop Sci. Biotech. 17 (3): 183-189.

Fan X, Cui F, Zhao C, Zhang W, Yang L, Zhao X, Han J, Su Q, Ji J, Zhao Z. (2015). QTLs for fag leaf size and their infuence on yield-related traits in wheat (Triticum aestivum L.). Mol. Breed. 35:24.

FAO STAT

(2020). http://www.fao.org/giews/countrybrief/country.jsp?c ode $=E G Y$.

Feltaous YM. (2019). Genetic diversity among some Egyptian bread wheat cultivars based on morphological characters and SSR markers. Assiut J. Agric. Sci. 50(4):35-50.

Giraldo P, Benavente E, Manzano-Agugliaro F, Gimenez E. (2019). Worldwide Research Trends on Wheat and Barley: A Bibliometric Comparative Analysis. Agronomy. 9: 352.

Ghasemi N, Mirfakhraii RG, Abbasi A. (2019). Assessment of Genetic Diversity of Bread Wheat (Triticum aestivum L.) Cultivars using Microsatellite Markers. jcb.; 11 (29) :9-16.

Gostimsky SA, Kokaeva ZG, Konovalov FA. (2005). Studying plant genome variation using molecular markers Russ. J. Genet. 41(4): 378-388.

Hassan MI. (2016). Assessment of Genetic Diversity in Bread Wheat Genotypes Based on Heat Tolerance and SSR Markers. Assiut J. Agric. Sci. 47 (5): 37-55. Hassan MI, Mohamed EA, El-Rawy MA, Amein KA. (2016). Evaluating Interspecific Wheat Hybrids Based on Heat and Drought Stress Tolerance. J. Crop Sci. Biotech. 19(1): 85-98.

Ijaz $U$ and Smiullah (2013). Heritability estimates of physiological and yield components in bread wheat under rainfed condition. International Journal of Modern Agriculture. 2(2): 58-62.

Jaiswal S, Sheoran S, Arora V, Angadi UB, Iquebal MA, Raghav N, Aneja B, Kumar D, Singh R, Sharma P, Singh GP, Rai A, Tiwari R, Kumar D. (2017). Putative Microsatellite DNA Marker-Based
Wheat Genomic Resource for Varietal Improvement and Management. Front. Plant Sci. 8: 1-10.

Jamali A, Sohrabi Y, Siose Mardeh A, Hoseinpanahi F. (2020). Morphological and yield responses of 20 genotypes of bread wheat to drought stress. Arch. Biol. Sci. 72: 71-9.

Jenks MA, Ashworth EN (1999). Plant epicuticular waxes: function, production, and genetics. Hortic. Rev. 23:1-68

Jensen NF, Driscoll CJ. (1962). Inheritance of the waxless character in wheat. Crop Sci. 2: 504-505.

Keller M, Karutz C, Schmid JE, Stamp P, Winzeler M, Keller B, Messmer MM. (1999). Quantitative trait loci for lodging resistance in segregating wheat $\mathrm{x}$ spelt population. Theor. Appl. Genet. 98: 1171:1182.

Khaled AGA, Motawea MH, Said AA. (2015). Identification of ISSR and RAPD markers linked to yield traits in bread wheat under normal and drought conditions, J. Genet. Eng. Biotechnol. 13: 243-252.

Khan, MK, Pandey A, Thomas G, Akkaya MS, Kayis SA, Ozsensoy Y, Hamurcu M, Gezgin S, Topal A, Hakki EE. (2015). Genetic diversity and population structure of wheat in India and Turkey. AoB PLANTS, 7 , https://doi.org/10.1093/aobpla/plv083.

Khodadadi M, Fotokian MH, Miransari M. (2011). Genetic diversity of wheat (Triticum aestivum L.) genotypes based on cluster and principal component analyses for breeding strategies. Aust. J. Crop Sci. 5(1):17-24.

Liu Y, Tao Y, Wang Z, Guo Q. (2018). Identification of QTL for flag leaf length in common wheat and their pleiotropic eff ects. Mol. Breeding 38: 11.

Malik R, Sharma H, Verma A, Kundu S, Sharma I, Chatrath R. (2013). Hierarchical clustering of Indian wheat varieties using morphological diversity assessment. Indian J. Agric. Res. 47(2): 116-123.

Malik R, Sharma H, Sharma I, Kundu S, Verma A, Sheoran S, Kumar R, Chatrath R. (2014). Genetic diversity of agro-morphological characters in Indian wheat varieties using GT biplot. A. J. C. S. 8(9):12661271

Mardi M, Naghavi M, Pirseyedi S, Kazemi Alamooti M, Rashidi Monfared S, Ahkami A, Omidbakhsh M, Alavi N, Salehi Shanjani P, Katsiotis A. (2011). Comparative assessment of SSAP, AFLP and SSR markers for evaluation of genetic diversity of durum wheat (Triticum turgidum L. var. durum). J Agric. Sci. Technol. 13: 905-920.

Murray MG, Thompson WF. (1980). Rapid isolation of high molecular weight plant DNA. Nucleic Acids Res. 8(19): 4321-4325. 
Neale DB, Savolainen O. (2004). Association genetics of complex traits in conifers. Trends Plant Sci. 9: 325330 .

Nyquist, WE. (1991). Estimation of heritability and prediction of selection response in plant populations. Crit. Rev. Plant Sci. 10: 235-322.

Petrovic S, Maric T, Cupic A, Rebekic I, Rukavina S. (2017). Assessment of molecular and phenotypic diversity among winter wheat cultivars. Genetika 49 (2): 583-598.

Ping Lu, Qin J, Wang G, Wang L, Wang Z, Wu Q • Xie J, Liang Y, Wang Y, Zhang D, Sun O, Liu Z. (2015). Comparative fine mapping of the Wax 1 (W1) locus in hexaploid wheat. Theor. Appl. Genet. 128:1595-1603.

Powell W, Orozco-Castillo C, Chalmers KJ, Provan J, Waugh R. (1995). Polymerase chain reaction-based assays for the characterisation of plant genetic resources. Electrophoresis 16: 1726-1730.

Powell WW, Koput K, Smith-Doerr L. (1996). Interorganization collaboration and the locus of innovation: Networks of learning in biotechnology. Admin. Sci. Quart. 41: 116-145.

Prasad M, Varshney RK, Roy JK, Balyan HS, Gupta PK. (2000). The use of microsatellites for detecting DNA polymorphism, genotype identification and genetic diversity in wheat. Theor. Appl. Genet. 100(3-4): 584-592.

Prevost A and Wilkinson M. (1999). A new system of comparing PCR primers applied to ISSR fingerprinting of potato cultivars. Theor. Appl. Genet. 98: 107-112.

Roldan-Ruiz I, Dendauw J, Van Bockstaele E, Depicker A, De Loose M. (2000). AFLP markers reveal high polymorphic rates in ryegrasses (Lolium spp.). Mol. Breeding 6: 125-134.

Saleem B, Khan A, Shahzad M, Ijaz F. (2016). "Estimation of heritability and genetic advance for various metric traits in seven F2 populations of bread wheat (Triticum aestivum L.)," J. Agri. Sci., Belgrade, 61: 1-9.

Salem KF, Marion S, Borner RA. (2015). Assessing genetic diversity of Egyptian hexaploid wheat (Triticum aestivum L.) using microsatellite markers. Genet. Resour. Crop Evol. 62 (2): 377-385.

Sheoran S, Jaiswal S, Kumar D, Raghav N, Sharma R, Pawar S, Paul S, Iquebal MA, Jaiswar A, Sharma P, Singh R, Singh C, Gupta A, Kumar N, Angadi UP, Rai A, Singh GP, Kumar D, Tiwari R. (2019). Uncovering Genomic Regions Associated With 36 Agro-Morphological Traits in Indian Spring Wheat Using GWAS. Front. Plant Sci. (10): 1-20.

Thomelin P, Bonneau J, Brien C, Suchecki R, Baumann U, Kalambettu P, Langridge $P$,
Tricker P, Fleury D. (2019). The wheat Seven in Absentia gene is associated with increases in biomass and yield in hot climates. doi: https://doi.org/10.1101/726802.

Tsunewaki K, Ebana K. (1999). Production of nearisogenic lines of common wheat for glaucousness and genetic basis of this trait clarified by their use. Genes Genet. Syst. 74: 33-41.

Wang J, Luo MC, Chen Z, You FM, Wei Y, Zheng Y, Dvorak J. (2013). Aegilops tauschii single nucleotide polymorphisms shed light on the origins of wheat D-genome genetic diversity and pinpoint the geographic origin of hexaploid wheat. New Phytol. 198: 925-937.

Wei TM, Chang XP, Min DH, Jing RL. (2010). Analysis of genetic diversity and tapping elite alleles for plant height in drought-tolerant wheat varieties. Acta Agron. Sin. 36: 895-904.

Willick IR, Lahlali R. Vijayan P, Muir M, Karunakaran C, Tanino KK. (2018). Wheat flag leaf epicuticular wax morphology and composition in response to moderate drought stress are revealed by SEM, FTIR-ATR and synchrotron X-ray spectroscopy. Physio. Plantarum 162: 316-332.

Wu H, Qin J, Han J , Zhao X, Ouyang S, Liang Y, Zhang D, Wang Z, Wu Q, Xie J, Cui Y, Peng H, Sun Q, Liu Z. (2013). Comparative high-resolution mapping of the wax inhibitors Iw1 and Iw2 in hexaploid wheat. PLoS ONE 8 e84691.

Yang D, Liu Y, Cheng H, Chang L, Chen J, Chai S, Li M. (2016). Genetic dissection of fag leaf morphology in wheat (Triticum aestivum L.) under diverse water regimes. BMC Genet. 17: 94-102.

Zarkti H, Ouabbou H, Hilali A, Udupa SM. (2010). Detection of genetic diversity in Moroccan durum wheat accessions using agro-morphological traits and microsatellite markers. Afr. J. Agr. Res. 5:1837-1844. 
Mahmoud A. El-Rawy., 2020

\title{
الملخص العربي \\ تقييم التنوع الوراثي لبعض أصناف القمح المصري بناءً على الخصائص المورفولوجية \\ والواسمات الجزيئية (SSR marker)
}

\author{
محمود ابوالسعود الراوي محمد \\ استاذ مساعد- قسم الور اثذة ـ كلية الزر اعة ـ- جامعة اسيوط - اسيوط - مصر
}

يمكن أن تكون تقديرات التتوع الور اثي مفيدة في اختيار الطرز الجينية المهمة لمربي النباتات. في هذه الدراسة تم استخدام ثمانية أصناف من قمح المكرونة وثلاثة أصناف من فمح الخبز لدر اسة ومقارنة الصفات المورفولوجية مع الدراسة الجزيئية باستخدام تقدير ات التنوع الجيني المعتدة على تقنية SSR marker. لوحظت فروق معنوية وعالية المعنوية بين

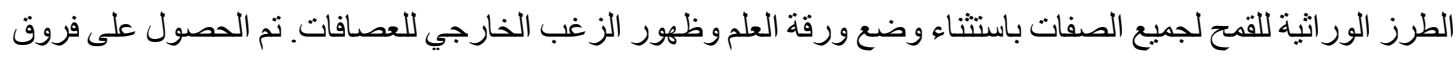

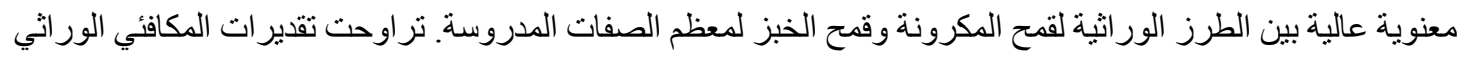

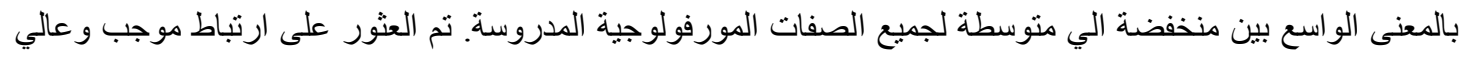

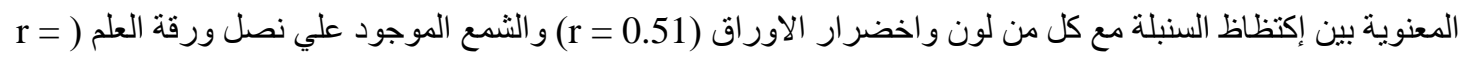
0.57) و الثمع الموجود علي السويقة (r = 0.60) وطول السويقة (r = r 0.53) ، بينما نم العثور على ارتباط سالب بين

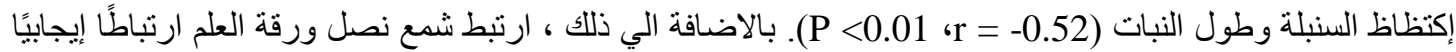

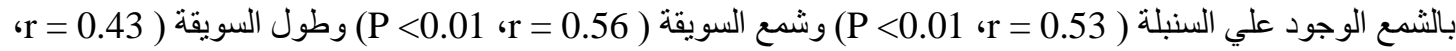

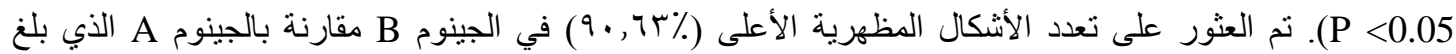

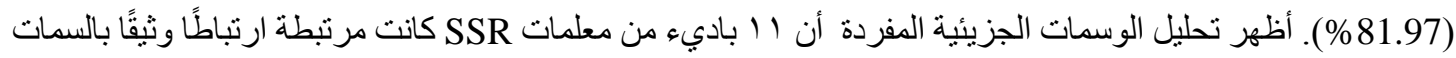

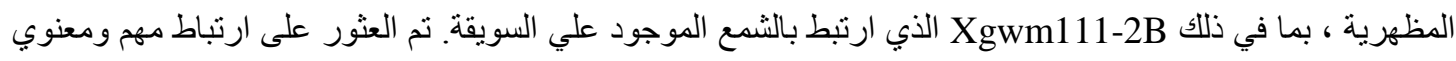

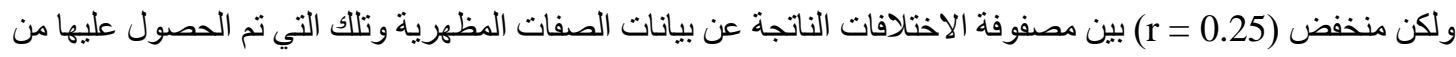

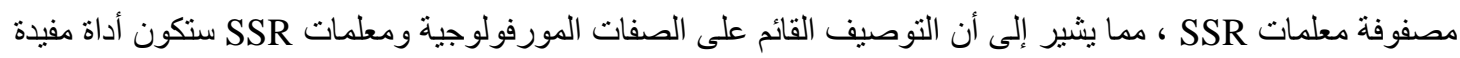
للمربين لاختيار الأنماط الجينية المناسبة. 